\title{
Regularly Varying Solutions of Second-Order Difference Equations with Arbitrary Sign Coefficient
}

\author{
Serena Matucci ${ }^{1}$ and Pavel Řehák $^{2}$ \\ ${ }^{1}$ Department of Electronics and Telecommunications, University of Florence, 50139 Florence, Italy \\ ${ }^{2}$ Institute of Mathematics, Academy of Sciences CR, Žižkova 22, 61662 Brno, Czech Republic \\ Correspondence should be addressed to Pavel Řehák, rehak@math.cas.cz
}

Received 15 June 2010; Accepted 25 October 2010

Academic Editor: E. Thandapani

Copyright (C) 2010 S. Matucci and P. Řehák. This is an open access article distributed under the Creative Commons Attribution License, which permits unrestricted use, distribution, and reproduction in any medium, provided the original work is properly cited.

Necessary and sufficient conditions for regular or slow variation of all positive solutions of a second-order linear difference equation with arbitrary sign coefficient are established. Relations with the so-called $\mathbb{M}$-classification are also analyzed and a generalization of the results to the halflinear case completes the paper.

\section{Introduction}

We consider the second-order linear difference equation

$$
\Delta^{2} y_{k}+p_{k} y_{k+1}=0
$$

on $\mathbb{N}$, where $p$ is an arbitrary sequence.

The principal aim of this paper is to study asymptotic behavior of positive solutions to (1.1) in the framework of discrete regular variation. Our results extend the existing ones for (1.1), see [1], where the additional condition $p_{k}<0$ was assumed. We point out that the relaxation of this condition requires a different approach. At the same time, our results can be seen as a discrete counterpart to the ones for linear differential equations, see, for example, [2]. As a byproduct, we obtain new nonoscillation criterion of Hille-Nehari type. We also examine relations with the so-called $\mathbb{M}$-classification (i.e., the classification of monotone solutions with respect to their limit behavior and the limit behavior of their difference). We point out that such relations could be established also in the continuous case, but, as far as we know, they have not been derived yet. In addition, we discuss relations with the sets of 
recessive and dominant solutions. A possible extension to the case of half-linear difference equations is also indicated.

The paper is organized as follows. In the next section we recall the concept of regularly varying sequences and mention some useful properties of (1.1) which are needed later. In the main section, that is, Section 3, we establish sufficient and necessary conditions guaranteeing that (1.1) has regularly varying solutions. Relations with the M-classification is analyzed in Section 4 . The paper is concluded by the section devoted to the generalization to the halflinear case.

\section{Preliminaries}

In this section we recall basic properties of regularly and slowly varying sequences and present some useful information concerning (1.1).

The theory of regularly varying sequences (sometimes called Karamata sequences), initiated by Karamata [3] in the thirties, received a fundamental contribution in the seventies with the papers by Seneta et al. (see $[4,5]$ ) starting from which quite many papers about regularly varying sequences have appeared, see [6] and the references therein. Here we make use of the following definition, which is a modification of the one given in [5], and is equivalent to the classical one, but it is more suitable for some applications to difference equations, see [6].

Definition 2.1. A positive sequence $y=\left\{y_{k}\right\}, k \in \mathbb{N}$, is said to be regularly varying of index $Q$, $\varrho \in \mathbb{R}$, if there exists $C>0$ and a positive sequence $\left\{\alpha_{k}\right\}$ such that

$$
\lim _{k} \frac{y_{k}}{\alpha_{k}}=C, \quad \lim _{k} k \frac{\Delta \alpha_{k}}{\alpha_{k}}=\rho .
$$

If $\rho=0$, then $\left\{y_{k}\right\}$ is said to be slowly varying. Let us denote by $R U(\rho)$ the totality of regularly varying sequences of index $\rho$ and by $\mathcal{S U}$ the totality of slowly varying sequences. A positive sequence $\left\{y_{k}\right\}$ is said to be normalized regularly varying of index $\rho$ if it satisfies $\lim _{k} k \Delta y_{k} / y_{k}=\rho$. If $\rho=0$, then $y$ is called a normalized slowly varying sequence. In the sequel, $\mathcal{N R V}(\rho)$ and $\mathcal{S S U}$ will denote, respectively, the set of all normalized regularly varying sequences of index $Q$, and the set of all normalized slowly varying sequences. For instance, the sequence $\left\{y_{k}\right\}=\{\log k\} \in \mathcal{N S V}$, and the sequence $\left\{y_{k}\right\}=\left\{k^{\rho} \log k\right\} \in \mathcal{N R V}(\rho)$, for every $\varrho \in \mathbb{R}$; on the other hand, the sequence $\left\{y_{k}\right\}=\left\{1+(-1)^{k} / k\right\} \in S \mathcal{N S V}$.

The main properties of regularly varying sequences, useful to the development of the theory in the subsequent sections, are listed in the following proposition. The proofs of the statements can be found in [1], see also [4,5].

Proposition 2.2. Regularly varying sequences have the following properties.

(i) A sequence $y \in \mathcal{R} V(\rho)$ if and only if $y_{k}=k^{\rho} \varphi_{k} \exp \left\{\sum_{j=1}^{k-1} \psi_{j} / j\right\}$, where $\left\{\varphi_{k}\right\}$ tends to a positive constant and $\left\{\psi_{k}\right\}$ tends to 0 as $k \rightarrow \infty$. Moreover, $y \in \mathcal{R} U(\rho)$ if and only if $y_{k}=k^{\rho} L_{k}$, where $L \in S \mathcal{S}$.

(ii) A sequence $y \in R U(\rho)$ if and only if $y_{k}=\varphi_{k} \prod_{j=1}^{k-1}\left(1+\delta_{j} / j\right)$, where $\left\{\varphi_{k}\right\}$ tends to a positive constant and $\left\{\delta_{k}\right\}$ tends to $\rho$ as $k \rightarrow \infty$.

(iii) If a sequence $y \in \mathcal{N R V}(\rho)$, then in the representation formulae given in (i) and (ii), it holds $\varphi_{k} \equiv$ const $>0$, and the representation is unique. Moreover, $y \in \mathcal{N R U}(\rho)$ if and only if $y_{k}=k^{\rho} S_{k}$, where $S \in \mathcal{N S V}$. 
(iv) Let $y \in R U(\rho)$. If one of the following conditions holds (a) $\Delta y_{k} \leq 0$ and $\Delta^{2} y_{k} \geq 0$, or (b) $\Delta y_{k} \geq 0$ and $\Delta^{2} y_{k} \leq 0$, or (c) $\Delta y_{k} \geq 0$ and $\Delta^{2} y_{k} \geq 0$, then $y \in \mathcal{N R U}(\rho)$.

(v) Let $y \in R U(\rho)$. Then $\lim _{k} y_{k} / k^{\rho-\varepsilon}=\infty$ and $\lim _{k} y_{k} / k^{\rho+\varepsilon}=0$ for every $\varepsilon>0$.

(vi) Let $u \in \mathcal{R} U\left(\rho_{1}\right)$ and $v \in \mathcal{R} U\left(\rho_{2}\right)$. Then $u v \in \mathcal{R} U\left(\rho_{1}+\rho_{2}\right)$ and $1 / u \in \mathcal{R} U\left(-\rho_{1}\right)$. The same holds if $R \circlearrowright$ is replaced by $N R U$.

(vii) If $y \in \mathcal{R} U(\rho), \rho \in \mathbb{R}$, is strictly convex, that is, $\Delta^{2} y_{k}>0$ for every $k \in \mathbb{N}$, then $y$ is decreasing provided $\rho \leq 0$, and it is increasing provided $\rho>0$. If $y \in \mathcal{R V}(\rho), \rho \in \mathbb{R}$, is strictly concave for every $k \in \mathbb{N}$, then $y$ is increasing and $\rho \geq 0$.

(viii) If $y \in R U(\rho)$, then $\lim _{k} y_{k+1} / y_{k}=1$.

Concerning (1.1), a nontrivial solution $y$ of (1.1) is called nonoscillatory if it is eventually of one sign, otherwise it is said to be oscillatory. As a consequence of the Sturm separation theorem, one solution of (1.1) is oscillatory if and only if every solution of (1.1) is oscillatory. Hence we can speak about oscillation or nonoscillation of equation (1.1). A classification of nonoscillatory solutions in case $p$ is eventually of one sign, will be recalled in Section 4. Nonoscillation of (1.1) can be characterized in terms of solvability of a Riccati difference equation; the methods based on this relation are referred to as the Riccati technique: equation (1.1) is nonoscillatory if and only if there is $a \in \mathbb{N}$ and a sequence $w$ satisfying

$$
\Delta w_{k}+p_{k}+\frac{w_{k}^{2}}{1+w_{k}}=0
$$

with $1+w_{k}>0$ for $k \geq a$. Note that, dealing with nonoscillatory solutions of (1.1), we may restrict our considerations just to eventually positive solutions without loss of generality.

We end this section recalling the definition of recessive solution of (1.1). Assume that (1.1) is nonoscillatory. A solution $y$ of (1.1) is said to be a recessive solution if for any other solution $x$ of (1.1), with $x \neq \lambda y, \lambda \in \mathbb{R}$, it holds $\lim _{k} y_{k} / x_{k}=0$. Recessive solutions are uniquely determined up to a constant factor, and any other linearly independent solution is called a dominant solution. Let $y$ be a solution of (1.1), positive for $k \geq a \geq 0$. The following characterization holds: $y$ is recessive if and only if $\sum_{k=a}^{\infty} 1 /\left(y_{k} y_{k+1}\right)=\infty ; y$ is dominant if and only if $\sum_{k=a}^{\infty} 1 /\left(y_{k} y_{k+1}\right)<\infty$.

\section{Regularly Varying Solutions of Linear Difference Equations}

In this section we prove conditions guaranteeing that (1.1) has regularly varying solutions. Hereinafter, $x_{k} \sim y_{k}$ means $\lim _{k} x_{k} / y_{k}=1$, where $x$ and $y$ are arbitrary positive sequences.

Let $A \in(-\infty, 1 / 4)$ and denote by $\rho_{1}<\rho_{2}$, the (real) roots of the quadratic equation $\rho^{2}-\rho+A=0$. Note that $1-2 \rho_{1}=\sqrt{1-4 A}>0,1-\rho_{1}=\varrho_{2}, \operatorname{sgn} A=\operatorname{sgn} \rho_{1}$, and $\rho_{2}>0$.

Theorem 3.1. Equation (1.1) is nonoscillatory and has a fundamental system of solutions $\{y, x\}$ such that $y_{k}=k^{\rho_{1}} L_{k} \in \mathcal{N R V}\left(\rho_{1}\right)$ and $x_{k}=k^{Q_{2}} \widetilde{L}_{k} \in \mathcal{N R V}\left(\rho_{2}\right)$ if and only if

$$
\lim _{k} k \sum_{j=k}^{\infty} p_{j}=A \in\left(-\infty, \frac{1}{4}\right)
$$


where $L, \tilde{L} \in \mathcal{N S V}$ with $\widetilde{L}_{k} \sim 1 /\left(\left(1-2 \varrho_{1}\right) L_{k}\right)$ as $k \rightarrow \infty$. Moreover, $y$ is a recessive solution, $x$ is a dominant solution, and every eventually positive solution $z$ of (1.1) is normalized regularly varying, with $z \in \mathcal{N R U}\left(\rho_{1}\right) \cup \mathcal{N R V}\left(\rho_{2}\right)$.

Proof. First we show the last part of the statement. Let $\{x, y\}$ be a fundamental set of solutions of (1.1), with $y \in \mathcal{N R V}\left(\rho_{1}\right), x \in \mathcal{N R V}\left(\varrho_{2}\right)$, and let $z$ be an arbitrary solution of (1.1), with $z_{k}>0$ for $k$ sufficiently large. Since $y \in \mathcal{N R U}\left(\rho_{1}\right)$, it can be written as $y_{k}=k^{\rho_{1}} L_{k}$, where $L \in \mathcal{S S U}$, by Proposition 2.2. Then $y_{k} y_{k+1}=k^{\rho_{1}}(k+1)^{\rho_{1}} L_{k} L_{k+1} \sim k^{2 \rho_{1}} L_{k}^{2}$ as $k \rightarrow \infty$. By Proposition 2.2, $L^{2} \in \mathcal{N S V}$, and $L_{k}^{2} k^{2 \rho_{1}-1} \rightarrow 0$ as $k \rightarrow \infty$, being $2 Q_{1}-1<0$. Hence, there is $N>0$ such that $L_{k}^{2} k^{2 \rho_{1}-1} \leq N$ for $k \geq a$, and

$$
\sum_{j=a}^{k} \frac{1}{y_{j} y_{j+1}} \sim \sum_{j=a}^{k} \frac{1}{j^{2 \rho_{1}} L_{j}^{2}} \geq \frac{1}{N} \sum_{j=a}^{k} \frac{1}{j} \longrightarrow \infty
$$

as $k \rightarrow \infty$. This shows that $y$ is a recessive solution of (1.1). Clearly, $x \in \mathcal{N R V}\left(\varrho_{2}\right)$ is a dominant solution, and $\lim _{k} y_{k} / x_{k}=0$. Now, let $c_{1}, c_{2} \in \mathbb{R}$ be such that $z=c_{1} y+c_{2} x$. Since $z$ is eventually positive, if $c_{2}=0$, then necessarily $c_{1}>0$ and $z \in \mathcal{N R V}\left(\rho_{1}\right)$. If $c_{2} \neq 0$, then we get $c_{2}>0$ because of the positivity of $z_{k}$ for $k$ large and the strict inequality between the indices of regular variation $\rho_{1}<Q_{2}$. Moreover, $z \in \mathcal{N R V}\left(\rho_{2}\right)$. Indeed, taking into account that $y_{k} / x_{k} \rightarrow 0, k \Delta y_{k} / y_{k} \rightarrow \rho_{1}$, and $k \Delta x_{k} / x_{k} \rightarrow \varrho_{2}$, it results

$$
\frac{k \Delta z_{k}}{z_{k}}=\frac{c_{1} k \Delta y_{k}+c_{2} k \Delta x_{k}}{c_{1} y_{k}+c_{2} x_{k}}=\frac{c_{1}\left(k \Delta y_{k} / y_{k}\right)\left(y_{k} / x_{k}\right)+c_{2} k \Delta x_{k} / x_{k}}{c_{1} y_{k} / x_{k}+c_{2}} \sim \frac{k \Delta x_{k}}{x_{k}} .
$$

Now we prove the main statement.

\section{Necessity}

Let $y \in \mathcal{N R V}\left(\rho_{1}\right)$ be a solution of (1.1) positive for $k \geq a$. Set $w_{k}=\Delta y_{k} / y_{k}$. Then $\lim _{k} k w_{k}=$ $\rho_{1}, \lim _{k} w_{k}=0$, and for any $M>0,\left|w_{k}\right| \leq M / k$ provided $k$ is sufficiently large. Moreover, $w$ satisfies the Riccati difference equation (2.2) and $1+w_{k}>0$ for $k$ sufficiently large. Now we show that $\sum_{j=a}^{\infty} w_{j}^{2} /\left(1+w_{j}\right)$ converges. For any $\varepsilon \in(0,1)$ we have $1+w_{k} \geq 1-\varepsilon$ for large $k$, say $k \geq a$. Hence,

$$
\sum_{j=a}^{\infty} \frac{w_{j}^{2}}{1+w_{j}} \leq \frac{1}{1-\varepsilon} \sum_{j=a}^{\infty} w_{j}^{2} \leq \frac{M^{2}}{1-\varepsilon} \sum_{j=a}^{\infty} \frac{1}{j^{2}}<\infty .
$$

Summing now (2.2) from $k$ to $\infty$ we get

$$
w_{k}=\sum_{j=k}^{\infty} p_{j}+\sum_{j=k}^{\infty} \frac{w_{j}^{2}}{1+w_{j}}
$$


in particular we see that $\Sigma^{\infty} p_{j}$ converges. The discrete L'Hospital rule yields

$$
\lim _{k} \frac{\sum_{j=k}^{\infty} w_{j}^{2} /\left(1+w_{j}\right)}{1 / k}=\lim _{k} \frac{k(k+1) w_{k}^{2}}{1+w_{k}}=\rho_{1}^{2} .
$$

Hence, multiplying (3.5) by $k$ we get

$$
k \sum_{j=k}^{\infty} p_{j}=k w_{k}-k \sum_{j=k}^{\infty} \frac{w_{j}^{2}}{1+w_{j}} \longrightarrow \rho_{1}-\rho_{1}^{2}=A
$$

as $k \rightarrow \infty$, that is, (3.1) holds. The same approach shows that $x \in \mathcal{N R V}\left(\varrho_{2}\right)$ implies (3.1).

\section{Sufficiency}

First we prove the existence of a solution $y \in \mathcal{N} U\left(\rho_{1}\right)$ of (1.1). Set $\psi_{k}=k \sum_{j=k}^{\infty} p_{j}-A$. We look for a solution of (1.1) in the form

$$
y_{k}=\prod_{j=a}^{k-1}\left(1+\frac{Q_{1}+\psi_{j}+w_{j}}{j}\right)
$$

$k \geq a$, with some $a \in \mathbb{N}$. In order that $y$ is a (nonoscillatory) solution of (1.1), we need to determine $w$ in (3.8) in such a way that

$$
u_{k}=\frac{Q_{1}+\psi_{k}+w_{k}}{k}
$$

is a solution of the Riccati difference equation

$$
\Delta u_{k}+p_{k}+\frac{u_{k}^{2}}{1+u_{k}}=0
$$

satisfying $1+u_{k}>0$ for large $k$. If, moreover, $\lim _{k} w_{k}=0$, then $y \in \mathcal{N R V}\left(\rho_{1}\right)$ by Proposition 2.2. Expressing (3.10) in terms of $w$, in view of (3.9), we get

$$
\Delta w_{k}-\frac{\rho_{1}+w_{k}-A}{k}+\frac{(k+1)\left(\rho_{1}+\psi_{k}+w_{k}\right)^{2}}{k^{2}+k\left(\rho_{1}+\psi_{k}+w_{k}\right)}=0
$$

that is,

$$
\Delta w_{k}+w_{k} \frac{2 Q_{1}-1+2 \psi_{k}}{k}+\frac{w_{k}^{2}+\psi_{k}^{2}+2 \rho_{1} \psi_{k}}{k}+(G w)_{k}=0
$$


where $G$ is defined by

$$
(G w)_{k}=\frac{(k+1)\left(\rho_{1}+\psi_{k}+w_{k}\right)^{2}}{k^{2}+k\left(\rho_{1}+\psi_{k}+w_{k}\right)}-\frac{\left(\rho_{1}+\psi_{k}+w_{k}\right)^{2}}{k} .
$$

Introduce the auxiliary sequence

$$
h_{k}=\prod_{j=a}^{k-1}\left(1+\frac{2 \varrho_{1}-1+2 \psi_{j}}{j}\right)
$$

where $a$ sufficiently large will be determined later. Note that $h \in \mathcal{N R V}\left(2 \varrho_{1}-1\right)$ with $2 \varrho_{1}-1<$ 0 , hence $h_{k}$ is positively decreasing toward zero, see Proposition 2.2. It will be convenient to rewrite (3.12) in terms of $h$. Multiplying (3.12) by $h$ and using the identities $\Delta\left(h_{k} w_{k}\right)=$ $h_{k} \Delta w_{k}+\Delta h_{k} w_{k}+\Delta h_{k} \Delta w_{k}$ and $\Delta h_{k}=h_{k}\left(2 \varrho_{1}-1+2 \psi_{k}\right) / k$, we obtain

$$
\Delta\left(h_{k} w_{k}\right)+\frac{h_{k}}{k}\left(w_{k}^{2}+\psi_{k}^{2}+2 \varrho_{1} \psi_{k}\right)+h_{k}(G w)_{k}-\Delta h_{k} \Delta w_{k}=0
$$

If $h_{k} w_{k} \rightarrow 0$ as $k \rightarrow \infty$, summation of (3.15) from $k$ to $\infty$ yields

$$
w_{k}=\frac{1}{h_{k}} \sum_{j=k}^{\infty} \frac{h_{j}}{j}\left(w_{j}^{2}+\psi_{j}^{2}+2 \varrho_{1} \psi_{j}\right)+\frac{1}{h_{k}} \sum_{j=k}^{\infty} h_{j}(G w)_{j}-\frac{1}{h_{k}} \sum_{j=k}^{\infty} \Delta h_{j} \Delta w_{j} .
$$

Solvability of this equation will be examined by means of the contraction mapping theorem in the Banach space of sequences converging towards zero. The following properties of $h$ will play a crucial role in the proof. The first two are immediate consequences of the discrete L'Hospital rule and of the property of regular variation of $h$ :

$$
\begin{gathered}
\lim _{k} \frac{1}{h_{k}} \sum_{j=k}^{\infty} \frac{h_{j}}{j}=\frac{1}{1-2 \varrho_{1}}>0, \\
\lim _{k} \frac{1}{h_{k}} \sum_{j=k}^{\infty} \frac{h_{j}}{j} \alpha_{j}=0 \quad \text { provided } \lim _{k} \alpha_{k}=0 .
\end{gathered}
$$

Further we claim that

$$
\lim _{k} \frac{\sum_{j=k}^{\infty}\left|\Delta^{2} h_{j}\right|}{h_{k}}=0
$$

Indeed, first note that $\sum_{j=k}^{\infty}\left|\Delta h_{j}\right| \leq\left(1-2 \varrho_{1}+2 \sup _{j \geq k}\left|\psi_{j}\right|\right) \sum_{j=k}^{\infty} h_{j} / j<\infty$, and so $\sum_{j=k}^{\infty}\left|\Delta^{2} h_{j}\right| \leq$ $\sum_{j=k}^{\infty}\left(\left|\Delta h_{j}\right|+\left|\Delta h_{j+1}\right|\right)<\infty$. By the discrete L'Hospital rule we now have that

$$
\lim _{k} \frac{\sum_{j=k}^{\infty}\left|\Delta^{2} h_{j}\right|}{h_{k}}=\lim _{k}\left|\frac{\Delta^{2} h_{k}}{\Delta h_{k}}\right|=\lim _{k}\left|\frac{\Delta h_{k+1}}{\Delta h_{k}}-1\right|=0
$$




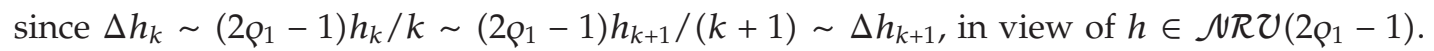
Denote $\widetilde{\psi}_{k}=\sup _{j \geq k}\left|\psi_{j}\right|$. Taking into account that $\lim _{k} \widetilde{\psi}_{k}=0$, and that (3.17) and (3.19) hold, it is possible to choose $\delta>0$ and $a \in \mathbb{N}$ in such a way that

$$
\begin{aligned}
& \frac{12 \delta}{1-2 \rho_{1}} \leq 1 \\
& \sup _{k \geq a} \frac{1}{h_{k}} \sum_{j=k}^{\infty} \frac{h_{j}}{j} \leq \frac{2}{1-2 \varrho_{1}} \\
& \widetilde{\psi}_{a}^{2}+2\left|\rho_{1}\right| \tilde{\psi}_{a} \leq \delta^{2}, \\
& \frac{\left(1+\left|\rho_{1}\right|+\tilde{\psi}_{a}+\delta\right)^{3}}{a-\left(\left|\rho_{1}\right|+\tilde{\psi}_{a}+\delta\right)} \leq \frac{\delta\left(1-2 \rho_{1}\right)}{6}, \\
& \sup _{k \geq a} \frac{1}{h_{k}} \sum_{j=k}^{\infty}\left|\Delta^{2} h_{j}\right| \leq \frac{1}{6} \\
& \frac{1-2 \varrho_{1}+2 \widetilde{\psi}_{a}}{a} \leq \frac{1}{6}, \\
& \gamma:=\frac{4 \delta}{1-2 \rho_{1}}+\frac{8\left(1+\left|\rho_{1}\right|+\widetilde{\psi}_{a}+\delta\right)^{2}}{1-2 \rho_{1}} \sup _{k \geq a} \frac{k+\left|\rho_{1}\right|+\tilde{\psi}_{a}+\delta}{\left(k-\left|\rho_{1}\right|-\widetilde{\psi}_{a}-\delta\right)^{2}} \\
& +\frac{1-2 \varrho_{1}+\tilde{\psi}_{a}}{a}+\sup _{k \geq a} \frac{1}{h_{k}} \sum_{j=k}^{\infty}\left|\Delta^{2} h_{j}\right|<1 .
\end{aligned}
$$

Let $\ell_{0}^{\infty}(a)$ be the Banach space of all the sequences defined on $\{a, a+1, \ldots\}$ and converging to zero, endowed with the sup norm. Let $\Omega$ denote the set

$$
\Omega=\left\{w \in \ell_{0}^{\infty}:\left|w_{k}\right| \leq \delta, k \geq a\right\}
$$

and define the operator $\tau$ by

$$
(\tau w)_{k}=\frac{1}{h_{k}} \sum_{j=k}^{\infty} \frac{h_{j}}{j}\left(w_{j}^{2}+\psi_{j}^{2}+2 \varrho_{1} \psi_{j}\right)+\frac{1}{h_{k}} \sum_{j=k}^{\infty} h_{j}(G w)_{j}-\frac{1}{h_{k}} \sum_{j=k}^{\infty} \Delta h_{j} \Delta w_{j},
$$

$k \geq a$. First we show that $\tau \Omega \subseteq \Omega$. Assume that $w \in \Omega$. Then $\left|(\tau w)_{k}\right| \leq K_{k}^{[1]}+K_{k}^{[2]}+K_{k}^{[3]}$, where $K_{k}^{[1]}=\left|\left(1 / h_{k}\right) \sum_{j=k}^{\infty}\left(h_{j} / j\right)\left(w_{j}^{2}+\psi_{j}^{2}+2 \rho_{1} \psi_{j}\right)\right|, K_{k}^{[2]}=\left|\left(1 / h_{k}\right) \sum_{j=k}^{\infty} h_{j}(G w)_{j}\right|$, and $K_{k}^{[3]}=$ $\left|\left(1 / h_{k}\right) \sum_{j=k}^{\infty} \Delta h_{j} \Delta w_{j}\right|$. In view of (3.21), (3.22), and (3.23), we have

$$
K_{k}^{[1]} \leq\left(\delta^{2}+\widetilde{\psi}_{a}^{2}+2\left|\varrho_{1}\right| \widetilde{\psi}_{a}\right) \frac{1}{h_{k}} \sum_{j=k}^{\infty} \frac{h_{j}}{j} \leq \frac{2\left(\delta^{2}+\widetilde{\psi}_{a}^{2}+2\left|\rho_{1}\right| \widetilde{\psi}_{a}\right)}{1-2 \varrho_{1}} \leq \frac{4 \delta^{2}}{1-2 \varrho_{1}} \leq \frac{\delta}{3},
$$


$k \geq a$. Thanks to (3.22) and (3.24), we get

$$
\begin{aligned}
K_{k}^{[2]} & \leq \frac{1}{h_{k}} \sum_{j=k}^{\infty} \frac{h_{j}}{j}\left|j(G w)_{j}\right| \leq \frac{1}{h_{k}} \sum_{j=k}^{\infty} \frac{h_{j}}{j} \cdot \frac{\left(1+\left|\varrho_{1}\right|+\widetilde{\psi}_{a}+\delta\right)^{3}}{j-\left(\left|\rho_{1}\right|+\widetilde{\psi}_{a}+\delta\right)} \\
& \leq \frac{\left(1+\left|\varrho_{1}\right|+\widetilde{\psi}_{a}+\delta\right)^{3}}{a-\left(\left|\rho_{1}\right|+\widetilde{\psi}_{a}+\delta\right)} \cdot \frac{2}{1-2 \varrho_{1}} \leq \frac{\delta}{3}
\end{aligned}
$$

$k \geq a$. Finally, summation by parts, (3.25), and (3.26) yield

$$
\begin{aligned}
K_{k}^{[3]} & =\left|\frac{1}{h_{k}} \lim _{t \rightarrow \infty}\left[w_{j} \Delta h_{j}\right]_{j=k}^{t}-\frac{1}{h_{k}} \sum_{j=k}^{\infty} \Delta^{2} h_{j} w_{j+1}\right| \\
& \leq\left|\frac{2 Q_{1}-1+2 \psi_{k}}{k} w_{k}\right|+\delta \frac{1}{h_{k}} \sum_{j=k}^{\infty}\left|\Delta^{2} h_{j}\right| \\
& \leq \frac{1-2 \varrho_{1}+2 \widetilde{\psi}_{a}}{a} \delta+\frac{\delta}{6} \leq \frac{\delta}{3},
\end{aligned}
$$

$k \geq a$. Hence, $\left|(\tau w)_{k}\right| \leq \delta, k \geq a$. Next we prove that $\lim _{k}(\tau w)_{k}=0$. Since $\lim _{k}\left(w_{k}^{2}+\psi_{k}^{2}+\right.$ $\left.2 \psi_{k}\right)=0$, we have $\lim _{k} K_{k}^{[1]}=0$ by (3.18). Since $\lim _{k}\left(1+\left|\rho_{1}\right|+\tilde{\psi}_{a}+\delta\right)^{3} /\left(k-\left(\left|\rho_{1}\right|+\tilde{\psi}_{a}+\delta\right)\right)=0$, we have $\lim _{k} K_{k}^{[2]}=0$ by (3.18). Finally, the discrete L'Hospital rule yields

$$
\lim _{k} \frac{\sum_{j=k}^{\infty} \Delta h_{j} \Delta w_{j}}{h_{k}}=\lim _{k}\left(-\Delta w_{k}\right)=0
$$

and $\lim _{k} K_{k}^{[3]}=0$. Altogether we get $\lim _{k}\left|(\tau w)_{k}\right|=0$, and so $\lim _{k}(\tau w)_{k}=0$. Hence, $\tau w \in \Omega$, which implies $\tau \Omega \subseteq \Omega$. Now we prove that $\tau$ is a contraction mapping on $\Omega$. Let $w, v \in \Omega$. Then, for $k \geq a,\left|(\tau w)_{k}-(\tau v)_{k}\right| \leq H_{k}^{[1]}+H_{k}^{[2]}+H_{k}^{[3]}$, where $H_{k}^{[1]}=\left|\left(1 / h_{k}\right) \sum_{j=k}^{\infty}\left(h_{j} / j\right)\left(w_{j}^{2}-v_{j}^{2}\right)\right|$, $H_{k}^{[2]}=\left|\left(1 / h_{k}\right) \sum_{j=k}^{\infty}\left(h_{j} / j\right)\left[(G w)_{j}-(G v)_{j}\right]\right|$, and $H_{k}^{[3]}=\left|\left(1 / h_{k}\right) \sum_{j=k}^{\infty} \Delta h_{j} \Delta\left(w_{j}-v_{j}\right)\right|$. In view of (3.22), we have

$$
\begin{aligned}
H_{k}^{[1]} & =\left|\frac{1}{h_{k}} \sum_{j=k}^{\infty} \frac{h_{j}}{j}\left(w_{j}-v_{j}\right)\left(w_{j}+v_{j}\right)\right| \\
& \leq\|w-v\| \frac{1}{h_{k}} \sum_{j=k}^{\infty} \frac{h_{j}}{j} 2 \delta \leq\|w-v\| \frac{4 \delta}{1-2 \varrho_{1}} .
\end{aligned}
$$


Before we estimate $H^{[2]}$, we need some auxiliary computations. The Lagrange mean value theorem yields $(G w)_{k}-(G v)_{k}=\left(w_{k}-v_{k}\right)((\partial G / \partial x) \xi)_{k}$, where $\min \left\{v_{k}, w_{k}\right\} \leq \xi_{k} \leq$ $\max \left\{v_{k}, w_{k}\right\}$ for $k \geq a$. Since

$$
\left|k\left(\frac{\partial G}{\partial x} \xi\right)_{k}\right| \leq \sup _{k \geq a} \frac{4\left(1+\left|\varrho_{1}\right|+\widetilde{\psi}_{a}+\delta\right)^{2}\left(k+\left|\varrho_{1}\right|+\tilde{\psi}_{a}+\delta\right)}{\left(k-\left|\rho_{1}\right|-\tilde{\psi}_{a}-\delta\right)^{2}}=: \gamma_{2},
$$

then, in view of (3.22),

$$
H_{k}^{[2]} \leq \gamma_{2}\|w-v\| \frac{1}{h_{k}} \sum_{j=k}^{\infty} \frac{h_{j}}{j} \leq\|w-v\| \frac{2 \gamma_{2}}{1-2 \varrho_{1}}
$$

$k \geq a$. Finally, using summation by parts, we get

$$
\begin{aligned}
H_{k}^{[3]} & =\left|\frac{1}{h_{k}} \lim _{t \rightarrow \infty}\left[\Delta h_{j}\left(w_{j}-v_{j}\right)\right]_{j=k}^{t}-\frac{1}{h_{k}} \sum_{j=k}^{\infty}\left(w_{j+1}-v_{j+1}\right) \Delta^{2} h_{j}\right| \\
& \leq\|w-v\|\left|\frac{\Delta h_{k}}{h_{k}}\right|+\|w-v\| \frac{1}{h_{k}} \sum_{j=k}^{\infty}\left|\Delta^{2} h_{j}\right| \leq \gamma_{3}\|w-v\|,
\end{aligned}
$$

$k \geq a$, where

$$
r_{3}:=\frac{1-2 Q_{1}+\tilde{\psi}_{a}}{a}+\sup _{k \geq a} \frac{1}{h_{k}} \sum_{j=k}^{\infty}\left|\Delta^{2} h_{j}\right|
$$

Noting that for $\gamma$ defined in (3.27) it holds, $\gamma=4 \delta /\left(1-2 \rho_{1}\right)+2 \gamma_{2} /\left(1-2 \rho_{1}\right)+\gamma_{3}$, we get $\left|(\tau w)_{k}-(\tau v)_{k}\right| \leq \gamma\|w-v\|$ for $k \geq a$. This implies $\|\tau w-\tau v\| \leq \gamma\|w-v\|$, where $\gamma \in(0,1)$ by virtue of (3.27).

Now, thanks to the contraction mapping theorem, there exists a unique element $w \in \Omega$ such that $w=\tau w$. Thus $w$ is a solution of (3.16), and hence of (3.11), and is positively decreasing towards zero. Clearly, $u$ defined by (3.9) is such that $\lim _{k} u_{k}=0$ and therefore $1+u_{k}>0$ for large $k$. This implies that $y$ defined by (3.8) is a nonoscillatory (positive) solution of (1.1). Since $\lim _{k}\left(\rho_{1}+\psi_{k}+w_{k}\right)=\rho_{1}$, we get $y \in \mathcal{N} U\left(\rho_{1}\right)$, see Proposition 2.2. By the same proposition, $y$ can be written as $y_{k}=k^{\rho_{1}} L_{k}$, where $L \in \mathcal{N}$ U.

Next we show that for a linearly independent solution $x$ of (1.1) we get $x \in \mathcal{N R U}\left(\rho_{2}\right)$. A second linearly independent solution is given by $x_{k}=y_{k} \sum_{j=a}^{k-1} 1 /\left(y_{j} y_{j+1}\right)$. Put $z=1 / y^{2}$. Then $z \in \mathcal{N R U}\left(-2 \varrho_{1}\right)$ and $z_{k} \sim 1 /\left(y_{k} y_{k+1}\right)$ by Proposition 2.2. Taking into account that $y$ is recessive and $\lim _{k} k z_{k}=\infty$ being $2 \varrho_{1}<1$ (see Proposition 2.2), the discrete L'Hospital rule yields

$$
\begin{aligned}
\lim _{k} \frac{k / y_{k}}{x_{k}} & =\lim _{k} \frac{k z_{k}}{\sum_{j=a}^{k-1} 1 /\left(y_{j} y_{j+1}\right)}=\lim _{k} \frac{z_{k}+(k+1) \Delta z_{k}}{1 /\left(y_{k} y_{k+1}\right)} \\
& =\lim _{k}\left(1+\frac{(k+1) \Delta z_{k}}{z_{k}}\right)=1-2 \varrho_{1} .
\end{aligned}
$$


Hence, $\left(1-2 \varrho_{1}\right) x_{k} \sim k / y_{k}=k^{1-\rho_{1}} / L_{k}$, that is, $x_{k} \sim k^{1-\rho_{1}} \widetilde{L}_{k}$, where $\tilde{L}_{k}=1 /\left(\left(1-2 \rho_{1}\right) L_{k}\right)$. Since $\widetilde{L} \in \mathcal{N S U}$ by Proposition 2.2, we get $x \in R U\left(1-\rho_{1}\right)=R \mathcal{R}\left(\rho_{2}\right)$ by Proposition 2.2. It remains to show that $x$ is normalized. We have

$$
\begin{aligned}
\frac{k \Delta x_{k}}{x_{k}} & =\frac{k \Delta y_{k} \sum_{j=a}^{k-1} 1 /\left(y_{j} y_{j+1}\right)+k y_{k+1} /\left(y_{k} y_{k+1}\right)}{x_{k}} \\
& =\frac{k \Delta y_{k}}{y_{k}}+\frac{k}{x_{k} y_{k}} .
\end{aligned}
$$

Thanks to this identity, since $k \Delta y_{k} / y_{k} \sim \rho_{1}$ and $\left(k / x_{k} y_{k}\right) \sim 1-2 \rho_{1}$, we obtain $\lim _{k} k \Delta x_{k} / x_{k}=$ $1-\rho_{1}=\rho_{2}$, which implies $x \in \mathcal{N R U}\left(\rho_{2}\right)$.

Remark 3.2. (i) In the above proof, the contraction mapping theorem was used to construct a recessive solution $y \in \mathcal{N R U}\left(\rho_{1}\right)$. A dominant solution $x \in \mathcal{N R U}\left(\rho_{2}\right)$ resulted from $y$ by means of the known formula for linearly independent solutions. A suitable modification of the approach used for the recessive solution leads to the direct construction of a dominant solution $x \in \mathcal{N R V}\left(\rho_{2}\right)$. This can be useful, for instance, in the half-linear case, where we do not have a formula for linearly independent solutions, see Section 5 .

(ii) A closer examination of the proof of Theorem 3.1 shows that we have proved a slightly stronger result. Indeed, it results

$$
y \in \operatorname{NRV}\left(\rho_{1}\right) \Longleftrightarrow \lim _{k} k \sum_{j=k}^{\infty} p_{j}=A<\frac{1}{4} \Longleftrightarrow x \in \operatorname{NRV}\left(\rho_{2}\right)
$$

Theorem 3.1 can be seen as an extension of [1, Theorems 1 and 2] in which $p$ is assumed to be a negative sequence, or as a discrete counterpart of [2, Theorems 1.10 and 1.11], see also [7, Theorem 2.3].

As a direct consequence of Theorem 3.1 we have obtained the following new nonoscillation criterion.

Corollary 3.3. If there exists the limit

$$
\lim _{k} k \sum_{j=k}^{\infty} p_{j} \in\left(-\infty, \frac{1}{4}\right)
$$

then (1.1) is nonoscillatory.

Remark 3.4. In [8] it was proved that, if

$$
-\frac{3}{4}<\liminf _{k} k \sum_{j=k}^{\infty} p_{j} \leq \underset{k}{\limsup } k \sum_{j=k}^{\infty} p_{j}<\frac{1}{4}
$$

then (1.1) is nonoscillatory. Corollary 3.3 extends this result in case $\lim _{k} k \sum_{j=k}^{\infty} p_{j}$ exists. 


\section{Relations with $\mathbb{M}$-Classification}

Throughout this section we assume that $p$ is eventually of one sign. In this case, all nonoscillatory solutions of (1.1) are eventually monotone, together with their first difference, and therefore can be a priori classified according to their monotonicity and to the values of the limits at infinity of themselves and of their first difference. A classification of this kind is sometimes called M-classification, see, for example, [9-12] for a complete treatment including more general equations. The aim of this section is to analyze the relations between the classification of the eventually positive solutions according to their regularly varying behavior, and the $\mathbb{M}$-classification. The relations with the set of recessive solutions and the set of dominant solutions will be also discussed. We point out that all the results in this section could be established also in the continuous case and, as far as we know, have never been derived both in the discrete and in the continuous case.

Because of linearity, without loss of generality, we consider only eventually positive solutions of (1.1). Since the situation differs depending on the sign of $p_{k}$, we treat separately the two cases. Note that (1.1), with $p$ negative, has already been investigated in [1], and therefore here we limit ourselves to state the main results, for the sake of completeness.

(I) $p_{k}>0$ for $k \geq a$

Any nonoscillatory solution $y$ of (1.1), in this case, satisfies $y_{k} \Delta y_{k}>0$ for large $k$, that is, all eventually positive solutions are increasing and concave. We denote this property by saying that $y$ is of class $\mathbb{M}^{+}$, being $\mathbb{M}^{+}=\left\{y: y\right.$ solution of (1.1), $y_{k}>0, \Delta y_{k}>0$ for large $\left.k\right\}$. This class can be divided in the subclasses

$$
\begin{aligned}
& \mathbb{M}_{\infty, B}^{+}=\left\{y \in \mathbb{M}^{+}: \lim _{k} y_{k}=\infty, \lim _{k} \Delta y_{k}=\ell_{y}, 0<\ell_{y}<\infty\right\}, \\
& \mathbb{M}_{\infty, 0}^{+}=\left\{y \in \mathbb{M}^{+}: \lim _{k} y_{k}=\infty, \lim _{k} \Delta y_{k}=0\right\}, \\
& \mathbb{M}_{B, 0}^{+}=\left\{y \in \mathbb{M}^{+}: \lim _{k} y_{k}=\ell_{y}, \lim _{k} \Delta y_{k}=0,0<\ell_{y}<\infty\right\}
\end{aligned}
$$

depending on the possible values of the limits of $y$ and of $\Delta y$. Solutions in $\mathbb{M}_{\infty, B}^{+}, \mathbb{M}_{\infty, 0}^{+}$, $\mathbb{M}_{B, 0}^{+}$are sometimes called, respectively, dominant solutions, intermediate solutions, and subdominant solutions, since, for large $k$, it holds $x_{k}>y_{k}>z_{k}$ for every $x \in \mathbb{M}_{\infty, B}^{+}, y \in \mathbb{M}_{\infty, 0}^{+}$, and $z \in \mathbb{M}_{B, 0}^{+}$. The existence of solutions in each subclass, is completely characterized by the convergence or the divergence of the series

$$
I=\sum_{k=a}^{\infty} k p_{k}
$$

see $[11,12]$. The following relations hold

$$
\begin{aligned}
& I<\infty \Longleftrightarrow \mathbb{M}^{+}=\mathbb{M}_{B, 0}^{+} \cup \mathbb{M}_{\infty, B}^{+} \text {, with } \mathbb{M}_{B, 0}^{+} \neq \emptyset, \mathbb{M}_{\infty, B}^{+} \neq \emptyset, \\
& I=\infty \text {, and (1.1) is nonoscillatory } \Longleftrightarrow \mathbb{M}^{+}=\mathbb{M}_{\infty, 0}^{+} \neq \emptyset .
\end{aligned}
$$


Let

$$
P=\lim _{k} k \sum_{j=k}^{\infty} p_{j}
$$

Since $k \sum_{j=k}^{\infty} p_{j}<\sum_{j=k}^{\infty} j p_{j}$, then the following relations between $I$ and $P$ hold:

(i) if $P>0$ then $I=\infty$;

(ii) if $I<\infty$ then $P=0$.

From Theorem 3.1, it follows that, if $P=0$, then (1.1) has a fundamental set of solutions $\{x, y\}$ with $x \in \mathcal{N S V}$, and $y \in \mathcal{N R V}(1)$; if $0<P<1 / 4$, then (1.1) has a fundamental set of solutions $\{u, v\}$ with $u \in \mathcal{N R U}\left(\rho_{1}\right)$, and $v \in \mathcal{N R U}\left(\rho_{2}\right), 0<\rho_{1}<\rho_{2}<1$. Further, all the positive

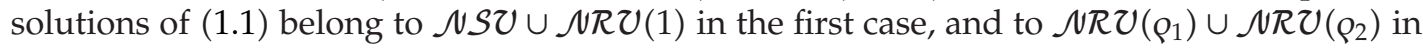
the second one. Set

$$
\begin{gathered}
\mathbb{M}_{S \mathcal{S U}}^{+}=\mathbb{M}^{+} \cap \mathcal{N S U}, \\
\mathbb{M}_{\text {RU }}^{+}(\rho)=\mathbb{M}^{+} \cap \mathcal{N R U}(\rho), \quad \rho>0 .
\end{gathered}
$$

By means of the above notation, the results proved in Theorem 3.1 can be summarized as follows

$$
\begin{aligned}
\emptyset \neq \mathbb{M}^{+}=\mathbb{M}_{\mathcal{~ S U}}^{+} \cup \mathbb{M}_{\mathcal{R V}}^{+}(1) & \Longleftrightarrow P=0, \\
\emptyset \neq \mathbb{M}^{+}=\mathbb{M}_{\mathcal{R U}}^{+}\left(\rho_{1}\right) \cup \mathbb{M}_{\mathcal{R U}}^{+}\left(\rho_{2}\right) & \Longleftrightarrow P \in\left(0, \frac{1}{4}\right) .
\end{aligned}
$$

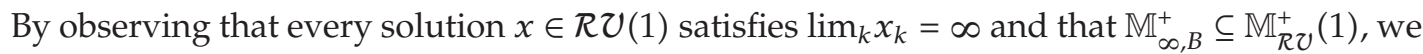
get the following result.

Theorem 4.1. For (1.1), with $p_{k}>0$ for large $k$, the following hold.

(i) If $P=0$ and $I<\infty$, then $\mathbb{M}^{+}=\mathbb{M}_{\mathcal{S U}}^{+} \cup \mathbb{M}_{\mathcal{R V}}^{+}$(1), with $\mathbb{M}_{\mathcal{S U}}^{+}=\mathbb{M}_{B, 0^{\prime}}^{+}, \mathbb{M}_{\mathcal{R V}}^{+}(1)=\mathbb{M}_{\infty, B}^{+}$.

(ii) If $P=0$ and $I=\infty$, then $\mathbb{M}^{+}=\mathbb{M}_{S \mathcal{S}}^{+} \cup \mathbb{M}_{\mathcal{R} V}^{+}(1)=\mathbb{M}_{\infty, 0}^{+}$.

(iii) If $P \in(0,1 / 4)$ then $\mathbb{M}^{+}=\mathbb{M}_{R \mathcal{R}}^{+}\left(\rho_{1}\right) \cup \mathbb{M}_{\mathcal{R U}}^{+}\left(\rho_{2}\right)=\mathbb{M}_{\infty, 0}^{+}$.

The above theorem shows how the study of the regular variation of the solutions and the M-classification supplement each other to give an asymptotic description of nonoscillatory solutions. Indeed, for instance, in case (i) the M-classification gives the additional information that all slowly varying solutions tend to a positive constant, while all the regularly varying solutions of index 1 are asymptotic to a positive multiple of $k$. On the other hand, in the remaining two cases, the study of the regular variation of the solutions gives the additional information that the positive solutions, even if they are all diverging with first difference tending to zero, have two possible asymptotic behaviors, since they can be slowly varying or regularly varying with index 1 in case (ii), or regularly varying with two different indices in case (iii). This distinction between eventually positive solutions is particularly meaningful in the study of dominant and recessive solutions. Let 
$\mathfrak{R}$ denote the set of all positive recessive solutions of (1.1) and $\mathfrak{D}$ denote the set of all positive dominant solutions of (1.1). From Theorem 4.1, taking into account Theorem 3.1, the following characterization of recessive and dominant solution holds.

(i) If $P=0$ and $I<\infty$, then $\mathfrak{R}=\mathbb{M}_{\mathcal{S U}}^{+}=\mathbb{M}_{B, 0}^{+}$and $\mathfrak{D}=\mathbb{M}_{\mathcal{R V}}^{+}(1)=\mathbb{M}_{\infty, B}^{+}$.

(ii) If $P=0$ and $I=\infty$, then $\mathfrak{R}=\mathbb{M}_{\mathcal{S U}}^{+} \subset \mathbb{M}_{\infty, 0}^{+}$and $\mathfrak{D}=\mathbb{M}_{\mathcal{R U}}^{+}(1) \subset \mathbb{M}_{\infty, 0}^{+}$.

(iii) If $P \in(0,1 / 4)$ and $I=\infty$, then $\mathfrak{R}=\mathbb{M}_{\mathcal{R V}}^{+}\left(\rho_{1}\right) \subset \mathbb{M}_{\infty, 0}^{+}$and $\mathfrak{D}=\mathbb{M}_{\mathcal{R U}}^{+}\left(\rho_{2}\right) \subset \mathbb{M}_{\infty, 0}^{+}$.

(II) $p_{k}<0$ for $k \geq a$

In this case, completely analyzed in [1], any positive solution $y$ is either decreasing or eventually increasing. We say that $y$ is of class $\mathbb{M}^{+}$in the first case, of class $\mathbb{M}^{-}$in the second one. It is easy to verify that every $y \in \mathbb{M}^{+}$satisfies $\lim _{k} y_{k}=\infty$, and every $y \in \mathbb{M}^{-}$satisfies $\lim _{k} \Delta y_{k}=0$. Therefore the sets $\mathbb{M}^{+}$and $\mathbb{M}^{-}$can be divided into the following subclasses

$$
\begin{aligned}
\mathbb{M}_{\infty, B}^{+} & =\left\{y \in \mathbb{M}^{+}: \lim _{k} y_{k}=\infty, \lim _{k} \Delta y_{k}=\ell_{y}, 0<\ell_{y}<\infty\right\}, \\
\mathbb{M}_{\infty, \infty}^{+} & =\left\{y \in \mathbb{M}^{+}: \lim _{k} y_{k}=\infty, \lim _{k} \Delta y_{k}=\infty\right\}, \\
\mathbb{M}_{B, 0}^{-} & =\left\{y \in \mathbb{M}^{-}: \lim _{k} y_{k}=\ell_{y}, \lim _{k} \Delta y_{k}=0,0<\ell_{y}<\infty\right\}, \\
\mathbb{M}_{0,0}^{-} & =\left\{y \in \mathbb{M}^{-}: \lim _{k} y_{k}=0, \lim _{k} \Delta y_{k}=0\right\} .
\end{aligned}
$$

Also in this case, the existence of solutions of (1.1) in each subclass is completely described by the convergence or divergence of the series $I$ given by (4.2)

$$
\begin{aligned}
& \mathbb{M}^{+}=\mathbb{M}_{\infty, \infty}^{+} \Longleftrightarrow I=-\infty \Longleftrightarrow \mathbb{M}^{-}=\mathbb{M}_{0,0}^{-} \\
& \mathbb{M}^{+}=\mathbb{M}_{\infty, B}^{+} \Longleftrightarrow I>-\infty \Longleftrightarrow \mathbb{M}^{-}=\mathbb{M}_{B, 0}^{-}
\end{aligned}
$$

Let

$$
\begin{gathered}
\mathbb{M}_{S \mathcal{U}}^{-}=\mathbb{M}^{-} \cap \mathcal{N S U}, \quad \mathbb{M}_{\mathcal{R U}}^{-}\left(\rho_{1}\right)=\mathbb{M}^{-} \cap \mathcal{N R V}\left(\rho_{1}\right), \quad \rho_{1}<0, \\
\mathbb{M}_{R V}^{+}\left(\rho_{2}\right)=\mathbb{M}^{+} \cap \operatorname{NRV}\left(\rho_{2}\right), \quad \varphi_{2}>0 .
\end{gathered}
$$

Notice that, being $p_{k}$ negative for large $k$, it results $\rho_{1} \leq 0, Q_{2} \geq 1$. The following holds.

Theorem 4.2 (see [1]). For (1.1), with $p_{k}<0$ for large $k$, it results in what follows.

(i) If $P=0$ and $I>-\infty$, then $\mathbb{M}_{\infty, B}^{+}=\mathbb{M}^{+}=\mathbb{M}_{\mathbb{R V}}^{+}(1)$ and $\mathbb{M}_{B, 0}^{-}=\mathbb{M}^{-}=\mathbb{M}_{S \mho}^{-}$.

(ii) If $P=0$ and $I=-\infty$, then $\mathbb{M}_{\infty, \infty}^{+}=\mathbb{M}^{+}=\mathbb{M}_{\mathcal{R V}}^{+}(1)$ and $\mathbb{M}_{0,0}^{-}=\mathbb{M}^{-}=\mathbb{M}_{S \mho}^{-}$.

(iii) If $P \in(-\infty, 0)$, then $\mathbb{M}_{\infty, \infty}^{+}=\mathbb{M}^{+}=\mathbb{M}_{\mathcal{R} U}^{+}\left(\rho_{2}\right)$ and $\mathbb{M}_{0,0}^{-}=\mathbb{M}^{-}=\mathbb{M}_{\mathcal{R} \mho}^{-}\left(\rho_{1}\right)$. 
Relations between recessive/dominant solutions and regularly varying solutions can be easily derived from the previous theorem, see also [1]. We have the following.

(i) If $P=0$ and $I>-\infty$, then $\mathfrak{R}=\mathbb{M}_{S \mathcal{S U}}^{-}=\mathbb{M}_{B, 0}^{-}$, and $\mathfrak{D}=\mathbb{M}_{\mathfrak{R V}}^{+}(1)=\mathbb{M}_{\infty, B}^{+}$.

(ii) If $P=0$ and $I=-\infty$, then $\mathfrak{R}=\mathbb{M}_{\mathcal{S U}}^{-}=\mathbb{M}_{0,0}^{-}$, and $\mathfrak{D}=\mathbb{M}_{\mathcal{R U}}^{+}(1)=\mathbb{M}_{\infty, \infty}^{+}$.

(iii) If $P \in(-\infty, 0)$, then $\mathfrak{R}=\mathbb{M}_{\mathcal{R V}}^{-}\left(\rho_{1}\right)=\mathbb{M}_{0,0}^{-}$, and $\mathfrak{D}=\mathbb{M}_{\mathcal{R} V}^{+}\left(\rho_{2}\right)=\mathbb{M}_{\infty, \infty}^{+}$.

We end this section by remarking that in this case positive solutions are convex and therefore they can exhibit also a rapidly varying behavior, unlike the previous case in which positive solutions are concave. We address the reader interested in this subject to the paper [1], in which the properties of rapidly varying sequences are described and the existence of rapidly varying solutions of (1.1) is completely analyzed for the case $p_{k}<0$.

\section{Regularly Varying Solutions of Half-Linear Difference Equations}

In this short section we show how the results of Section 3 can be extended to half-linear difference equations of the form

$$
\Delta\left(\Phi\left(\Delta y_{k}\right)\right)+p_{k} \Phi\left(y_{k+1}\right)=0
$$

where $p: \mathbb{N} \rightarrow \mathbb{R}$ and $\Phi(u)=|u|^{\alpha-1} \operatorname{sgn} u, \alpha>1$, for every $u \in \mathbb{R}$. For basic information on qualitative theory of (5.1) see, for example, [13].

Let $A \in\left(-\infty,(1 / \alpha)((\alpha-1) / \alpha)^{\alpha-1}\right)$ and denote by $\rho_{1}<\rho_{2}$, the (real) roots of the equation $\mid \rho^{\alpha /(\alpha-1)}-\rho+A=0$. Note that $\operatorname{sgn} A=\operatorname{sgn} \varrho_{1}$ and $\Phi^{-1}\left(\varrho_{1}\right)<(\alpha-1) / \alpha<\Phi^{-1}\left(\rho_{2}\right)$.

Theorem 5.1. Equation (5.1) is nonoscillatory and has two solutions $y, x$ such that $y \in$ $\mathcal{N R U}\left(\Phi^{-1}\left(\rho_{1}\right)\right)$ and $x \in \mathcal{N R U}\left(\Phi^{-1}\left(\rho_{2}\right)\right)$ if and only if

$$
\lim _{k} k^{\alpha-1} \sum_{j=k}^{\infty} p_{j}=A \in\left(-\infty, \frac{1}{\alpha}\left(\frac{\alpha-1}{\alpha}\right)^{\alpha-1}\right) .
$$

Proof. The main idea of the proof is the analogous of the linear case, apart from some additional technical problems. We omit all the details, pointing out only the main differences.

\section{Necessity}

Set $w_{k}=\Phi\left(\Delta y_{k} / y_{k}\right)$, then $w$ satisfies the generalized Riccati equation

$$
\Delta w_{k}+p_{k}+w_{k}\left(1-\frac{1}{\Phi\left(1+\Phi^{-1}\left(w_{k}\right)\right)}\right)=0
$$

and $\lim _{k} k^{\alpha-1} w_{k}=\rho_{1}$. The proof can then proceed analogously to the linear case.

\section{Sufficiency}

The existence of both solutions $y \in \mathcal{N R U}\left(\Phi^{-1}\left(\rho_{1}\right)\right)$ and $x \in \mathcal{N R U}\left(\Phi^{-1}\left(\rho_{2}\right)\right)$ needs to be proved by a fixed-point approach, since in the half-linear case there is no reduction of order 
formula for computing a linearly independent solution. For instance, a solution $y$ can be searched in the form

$$
y_{k}=\prod_{j=a}^{k-1}\left(1+\Phi^{-1}\left(\frac{\varrho_{1}+\psi_{j}+v_{j}}{j^{\alpha-1}}\right)\right),
$$

(compare with (3.8)), where $\psi_{k}=k^{\alpha-1} \sum_{j=k}^{\infty} p_{j}-A$ and $v$ is such that $u_{k}=\left(\rho_{1}+\psi_{k}+v_{k}\right) / k^{\alpha-1}$ is a solution of (5.3). All the other details are left to the reader.

Remark 5.2. (i) Theorem 5.1 can be seen as an extension of [6, Theorem 1] in which $p$ is assumed to be a negative sequence, and as a discrete counterpart of [14, Theorem 3.1].

(ii) A closer examination of the proof of Theorem 5.1 shows that we have proved a slightly stronger result which reads as follows:

$$
\begin{aligned}
y \in \mathcal{N R U}\left(\Phi^{-1}\left(\varrho_{1}\right)\right) & \Longleftrightarrow \lim _{k} k^{\alpha-1} \sum_{j=k}^{\infty} p_{j}=A<\frac{1}{\alpha}\left(\frac{\alpha-1}{\alpha}\right)^{\alpha-1} \\
& \Longleftrightarrow x \in \mathcal{N R V}\left(\Phi^{-1}\left(\varrho_{2}\right)\right) .
\end{aligned}
$$

Similarly as in the linear case, as a direct consequence of Theorem 5.1 we obtain the following new nonoscillation criterion. Recall that a Sturm type separation theorem holds for equation (5.1), see [13], hence one solution of (5.1) is nonoscillatory if and only if every solution of (5.1) is nonoscillatory.

Corollary 5.3. If there exists the limit

$$
\lim _{k} k^{\alpha-1} \sum_{j=k}^{\infty} p_{j} \in\left(-\infty, \frac{1}{\alpha}\left(\frac{\alpha-1}{\alpha}\right)^{\alpha-1}\right),
$$

then (5.1) is nonoscillatory.

\section{Acknowledgments}

This work was supported by the grants 201/10/1032 and 201/08/0469 of the Czech Grant Agency and by the Institutional Research Plan No. AV0Z10190503.

\section{References}

[1] S. Matucci and P. Ǩehák, "Second order linear difference equations and Karamata sequences," International Journal of Difference Equations, vol. 3, no. 2, pp. 277-288, 2008.

[2] V. Marić, Regular Variation and Differential Equations, vol. 1726 of Lecture Notes in Mathematics, Springer, Berlin, Germany, 2000.

[3] J. Karamata, "Sur certain "Tauberian theorems" de M. M. Hardy et Littlewood," Mathematica (Cluj) 3, pp. 33-48, 1930.

[4] R. Bojanić and E. Seneta, "A unified theory of regularly varying sequences," Mathematische Zeitschrift, vol. 134, pp. 91-106, 1973. 
[5] J. Galambos and E. Seneta, "Regularly varying sequences," Proceedings of the American Mathematical Society, vol. 41, pp. 110-116, 1973.

[6] S. Matucci and P. Řehák, "Regularly varying sequences and second order difference equations," Journal of Difference Equations and Applications, vol. 14, no. 1, pp. 17-30, 2008.

[7] J. Jaroš and T. Kusano, "Self-adjoint differential equations and generalized Karamata functions," Bulletin. Classe des Sciences Mathématiques et Naturelles, no. 29, pp. 25-60, 2004.

[8] P. Řehák, "Oscillation and nonoscillation criteria for second order linear difference equations," Fasciculi Mathematici, no. 31, pp. 71-89, 2001.

[9] M. Cecchi, Z. Došlá, and M. Marini, "Positive decreasing solutions of quasi-linear difference equations," Computers E Mathematics with Applications, vol. 42, no. 10-11, pp. 1401-1410, 2001.

[10] M. Cecchi, Z. Došlá, M. Marini, and I. Vrkoč, "Summation inequalities and half-linear difference equations," Journal of Mathematical Analysis and Applications, vol. 302, no. 1, pp. 1-13, 2005.

[11] M. Cecchi, Z. Došlá, and M. Marini, "On the growth of nonoscillatory solutions for difference equations with deviating argument," Advances in Difference Equations, Article ID 505324, 15 pages, 2008.

[12] M. Cecchi, Z. Došlá, and M. Marini, "On oscillation and nonoscillation properties of Emden-Fowler difference equations," Central European Journal of Mathematics, vol. 7, no. 2, pp. 322-334, 2009.

[13] P. Řehák, "Oscillatory properties of second order half-linear difference equations," Czechoslovak Mathematical Journal, vol. 51, no. 2, pp. 303-321, 2001.

[14] J. Jaroš, T. Kusano, and T. Tanigawa, "Nonoscillation theory for second order half-linear differential equations in the framework of regular variation," Results in Mathematics, vol. 43, no. 1-2, pp. 129-149, 2003. 